\title{
Highly Sensitive Silicon Nanowire Biosensor Devices for the Investigation of UniCAR Platform in Immunotherapy ${ }^{\dagger}$
}

\author{
Trang-Anh Nguyen-Le ${ }^{1, *}$, Diana Isabel Sandoval Bojorquez ${ }^{1} \mathbb{(}$, Arnau Pérez Roig ${ }^{2}$, Bergoi Ibarlucea ${ }^{3}{ }^{(0)}$, \\ Gianaurelio Cuniberti ${ }^{3}{ }^{-}$, Anja Feldmann ${ }^{1}$, Michael Bachmann ${ }^{1}\left(\mathbb{D}\right.$ and Larysa Baraban ${ }^{1}$ \\ 1 Institute of Radiopharmaceutical Cancer Research, Helmholtz-Zentrum Dresden-Rossendorf, \\ 01328 Dresden, Germany; d.sandoval-bojorquez@hzdr.de (D.I.S.B.); a.feldmann@hzdr.de (A.F.); \\ m.bachmann@hzdr.de (M.B.); larysa.baraban@tu-dresden.de (L.B.) \\ 2 Biotechnology Center, Technische Universität Dresden, 01307 Dresden, Germany; arnauperezroig@gmail.com \\ 3 Institute of Materials Science, Max Bergmann Center for Biomaterials, Technische Universität Dresden, \\ 01069 Dresden, Germany; bergoi.ibarlucea@tu-dresden.de (B.I.); Gianaurelio.Cuniberti@tu-dresden.de (G.C.) \\ * Correspondence: t.nguyen-le@hzdr.de \\ + Presented at the 8th International Symposium on Sensor Science, 17-28 May 2021; Available online: \\ https://i3s2021dresden.sciforum.net/.
}

check for

updates

Citation: Nguyen-Le, T.-A.;

Bojorquez, D.I.S.; Roig, A.P.;

Ibarlucea, B.; Cuniberti, G.; Feldmann,

A.; Bachmann, M.; Baraban, L. Highly Sensitive Silicon Nanowire Biosensor Devices for the Investigation of UniCAR Platform in Immunotherapy. Eng. Proc. 2021, 6, 20. https://doi.org/ 10.3390/I3S2021Dresden-10109

Academic Editors: Gianaurelio

Cuniberti and Larysa Baraban

Published: 17 May 2021

Publisher's Note: MDPI stays neutral with regard to jurisdictional claims in published maps and institutional affiliations.

Copyright: (c) 2021 by the authors. Licensee MDPI, Basel, Switzerland. This article is an open access article distributed under the terms and conditions of the Creative Commons Attribution (CC BY) license (https:/ / creativecommons.org/licenses/by/ $4.0 /)$.

\begin{abstract}
Although showing impressive therapeutic potential, treatments of leukemias with T-cells expressing chimeric antigen receptors (CARs) is limited by their risk of several severe side effects. To overcome these problems, a switchable CAR platform has been developed termed UniCAR. Unlike conventional CAR, which is directed against tumor-associated antigens, UniCAR treatment involves an intermediate target module (TM), which can cross-link UniCAR T cells with tumor cells and lead to destruction. The development of these novel TMs against different tumor targets requires numerous repetitive tests on different synthesizing trials, which is usually limited in quantity and time-consuming. Meanwhile, nano-biosensors are lately known as analytical tools, which are highly sensitive, label-free, rapid and reagent-saving. Among them, silicon nanowire (SiNW) sensors have been extensively investigated by researchers over the past decades thanks to their compatibility with CMOS technology, enabling mass production. In this work, we demonstrated the application of a previously published SiNW biosensor on the detection of the binding of UniCAR and a part of different TMs. The results underline the advantages of the SiNW sensor over the ELISA method in terms of ease of preparation, speed and sensitivity. The method is able to evaluate the binding affinity of UniCAR to different TMs and open a potential to quantify the number of active UniCAR T-cells in an in vivo sample at a later stage. In the end, the application of a nanosensor may speed up the R\&D process of the UniCAR concept and later play an important role in clinical monitoring of immunotherapy, especially in the era of precision medicine.
\end{abstract}

Keywords: biosensor; immunotherapy

Conflicts of Interest: The authors declare no conflict of interest. 